\title{
Host specialization of the blast fungus Magnaporthe oryzae is associated with dynamic gain and loss of genes linked to transposable elements
}

Kentaro Yoshida ${ }^{1,2,3^{*}}$, Diane G. O. Saunders ${ }^{4,5}$, Chikako Mitsuoka', Satoshi Natsume ${ }^{1}$, Shunichi Kosugi ${ }^{1}$, Hiromasa Saitoh?', Yoshihiro Inoue ${ }^{3}$, Izumi Chuma ${ }^{3}$, Yukio Tosa ${ }^{3}$, Liliana M. Cano ${ }^{2,6}$, Sophien Kamoun² and Ryohei Terauchi ${ }^{1 *}$

\begin{abstract}
Background: Magnaporthe oryzae (anamorph Pyricularia oryzae) is the causal agent of blast disease of Poaceae crops and their wild relatives. To understand the genetic mechanisms that drive host specialization of M. oryzae, we carried out whole genome resequencing of four $M$. oryzae isolates from rice (Oryza sativa), one from foxtail millet (Setaria italica), three from wild foxtail millet $S$. viridis, and one isolate each from finger millet (Eleusine coracana), wheat (Triticum aestivum) and oat (Avena sativa), in addition to an isolate of a sister species M. grisea, that infects the wild grass Digitaria sanguinalis.

Results: Whole genome sequence comparison confirmed that M. oryzae Oryza and Setaria isolates form a monophyletic and close to another monophyletic group consisting of isolates from Triticum and Avena. This supports previous phylogenetic analysis based on a small number of genes and molecular markers. When comparing the host specific subgroups, $1.2-3.5 \%$ of genes showed presence/absence polymorphisms and 0-6.5 \% showed an excess of non-synonymous substitutions. Most of these genes encoded proteins whose functional domains are present in multiple copies in each genome. Therefore, the deleterious effects of these mutations could potentially be compensated by functional redundancy. Unlike the accumulation of nonsynonymous nucleotide substitutions, gene loss appeared to be independent of divergence time. Interestingly, the loss and gain of genes in pathogens from the Oryza and Setaria infecting lineages occurred more frequently when compared to those infecting Triticum and Avena even though the genetic distance between Oryza and Setaria lineages was smaller than that between Triticum and Avena lineages. In addition, genes showing gain/loss and nucleotide polymorphisms are linked to transposable elements highlighting the relationship between genome position and gene evolution in this pathogen species.
\end{abstract}

Conclusion: Our comparative genomics analyses of host-specific M. oryzae isolates revealed gain and loss of genes as a major evolutionary mechanism driving specialization to Oryza and Setaria. Transposable elements appear to facilitate gene evolution possibly by enhancing chromosomal rearrangements and other forms of genetic variation.

Keywords: Magnaporthe oryzae, Pathogenomics, Host specialization, Functional redundancy, Transposable elements, Evolution

\footnotetext{
* Correspondence: Kentaro.Yoshida@port.kobe-u.ac.jp; terauchi@ibrc.or.jp

${ }^{1}$ Iwate Biotechnology Research Center, Kitakami, Iwate, Japan

Full list of author information is available at the end of the article
} 


\section{Background}

Magnaporthe oryzae causes disease on an array of important crops such as rice, wheat, barley, finger millet, foxtail millet, and wild grasses [1]. $M$. oryzae has received considerable attention as the causal agent of rice blast disease [2], which leads to 10-30 \% loss of harvest per year [3]. M oryzae is categorized into several hostspecific subgroups that are pathogenic on a variety of plants that include Oryza spp., Setaria spp., Triticum spp., Avena spp., Eleusine spp. etc. [1, 4, 5]. As with some fungal pathogens $M$. oryzae reproduction is predominantly asexual. When environmental conditions are conducive, $M$. oryzae generates an abundance of asexual clones that rapidly colonize its host. The rapid spread of such asexual pathogens poses a significant threat to global food security, human health and biodiversity $[6,7]$.

Sexual reproduction for $M$. oryzae is limited to specific geographic regions such as Yunnan, China and India [810]. The shift from sexual to asexual reproduction is considered to drive an evolutionary impasse [11]. For instance, linkage between nucleotide positions at each chromosome prevents purifying selection from removing newly arising deleterious mutations. This elevates the accumulation of these deleterious mutations, thereby reducing fitness in a given environment. However, a recent comparative genomic study of asexual pathogens revealed chromosomal rearrangements as a major mechanism for host-specific adaptation [12]. In fungi and oomycete pathogens, these chromosomal rearrangements occur frequently in transposon-rich regions, lineage specific regions and sub-telomeric regions that are enriched for effector genes [13-16]. Effector proteins secreted by pathogens alter the physiology of host plants and enhance colonization by pathogens, often determining the success or failure of infection. Through chromosomal rearrangements effector genes can be gained or lost, diversifying the effector repertoire even in the absence of sexual reproduction $[12,17]$.

For $M$. oryzae, whole-genome comparative analysis between two Oryza isolates P131 (from Japan) and Y34 (from China) revealed frequent chromosomal rearrangements, resulting in structural variation such as gain and loss of genes [18]. The genomic location of the avirulence effector AVR-Pita was more variable among asexual rice infecting isolates compared with other cereal infecting isolates, suggesting that selection pressures acting from its cognate resistance gene Pita of rice may trigger the diversity in the genome location of AVR-Pita [15]. This observation evokes the possibility that the frequency of chromosomal rearrangements may somehow be associated with selection pressure exerted by resistant hosts. In addition, nucleotide substitution is also a potent mechanism for effector diversification [19, 20]. For instance, alleles of $A V R$-Pik that differ in just a few amino acids interact with different specificities with alleles of the resistance gene $\operatorname{Pik}[21,22]$.

To date, the evolutionary mechanisms that drive adaptation of $M$. oryzae to specific hosts remain unclear. It has been proposed that Oryza-specific isolates of $M$. oryzae are most closely related to those identified on Setaria [4]. Whereas the host shift/jump to rice for $M$. oryzae is considered to have happened less than 10,000 years ago following rice domestication [1], $M$. oryzae on Triticum was only discovered in Brazil in the late 20th century [23]. This time difference in host shift/ jump may have generated distinct patterns of genetic variation between these $M$. oryzae sub-populations. Almost all Oryza isolates apart from the center of origin propagate asexually and are only found on cultivated rice (O. sativa) [24]. This makes these isolates particularly vulnerable to artificial selection that resulted from breeding resistant rice cultivars as Oryza isolates cannot infect any other host plant. In contrast, M. oryzae isolates that are adapted to wild Setaria spp. have the same host range as isolates from cultivated foxtail millet $S$. italica [25], suggesting that Setaria isolates can escape the artificial selection of breeding by shifting to wild host species. This difference between Oryza and Setaria pathogens could influence their genetic variation within these host-adapted $M$. oryzae populations.

To investigate the evolutionary mechanisms that drive $M$. oryzae host adaptation, we reconstructed the genome sequences of representative isolates from $M$. oryzae specifically infecting Oryza, Setaria, Eleusine, Avena and Triticum plant species and its sister species $M$. grisea. Host species specificity in $M$. oryzae has been studied by genetic analyses of these representatives of $M$. oryzae [26-28]. In additions, we resequenced another three isolates each from Oryza and Setaria pathogens to examine genetic polymorphisms within population of these pathogens. Recently, Chiapello et al. 2015 [29] studied molecular evolution of $M$. oryzae host-specific subgroups. They revealed that most genes were conserved among M. oryzae host-specific subgroups, and only a small number of gene families was associated with host specificity [29]. Compared with their studies, we focused on differences in molecular evolutionary pattern between the host-specific subgroups (Oryza-Setaria vs. AvenaTriticum pathogens) using different approaches. We discovered that genes showing gain/loss and excess of nonsynonymous substitutions usually encoded proteins whose functional domains are present in multiple copies in each genome. We identified a higher degree of presence/absence polymorphisms for Oryza and Setaria infecting $M$. oryzae isolates when compared to isolates from Triticum and Avena even though the genetic distance between Oryza and Setaria pathogens was smaller than that between isolates from Triticum and Avena. We 
also found that the lysin motif (LysM) domain effector gene Secreted LysM protein 1 (Slp1) was lost in the tested Setaria isolates. These observations suggest that gain and loss of genes in M. oryzae may act as a major evolutionary mechanism driving specialization to Oryza and Setaria host species. We could also link genes showing presence/absence and nucleotide polymorphisms to transposable elements (TEs), suggesting that TEs may trigger chromosomal rearrangements and mutation.

\section{Results}

Genome assembly of $M$. oryzae host-specific subgroup species and $M$. grisea

We selected four M. oryzae Oryza isolates, four Setaria isolates, five host-specific subgroup species and its sister species M. grisea, a pathogen of Digitaria species for full genome sequencing (Additional file 1: Table S1). We performed de novo assembly for one representative from each host-specific subgroup of $M$. oryzae (Ina168, GFSI1-7-2, Br48, Br58 and Z2-1) and M. grisea Dig41 (Table 1). The total assembly size (38.0-40.7 Mbp), N50 $(64.1-97.5 \mathrm{Kbp})$ and number of predicted gene models $(12,363-12,671)$ of $M$. oryzae, were similar to those of previously sequenced genomes of M. oryzae 70-15 [2], P131 and Y34 [18], but were smaller than M. oryzae host-specifc subgroups in Chiapello et al. 2015 (13,57114,781) [29]. To further investigate the assembled gene space we performed CEGMA analysis (Additional file 1: Table S2) to assess the completeness of 458 core eukaryotic protein genes (CEGs) in each genome assembly [30]. We used the published genome sequences of M. oryzae 70-15 strain [2] and P131 isolate [18] for comparison. The 248 CEGs are classified into four groups [30]. Group1 indicates the least conserved genes of the
248 CEGs. Group number increases with the increasing degree of conservation. The percentage of CEGs for $M$. oryzae host-specific isolates excluding $\mathrm{Br} 48$ was consistent with that of M. oryzae 70-15 and P131. However, the $M$. oryzae Br48 and M. grisea Dig41 isolates showed a lower percentage of CEGs in Group1 and Group3 compared with the controls although these percentages were still comparable to those reported for other published fungal genome sequences [31, 32].

To classify orthologous and paralogous relationships among the gene models of $M$. oryzae and M. grisea isolates, we performed OrthoMCL analysis [33] on all $M$. oryzae isolates and M. grisea Dig41 in addition to the published gene models of 70-15 strain, Y34 isolate and P131 isolate. We obtained 13,274 orthogroups, 7845 of which are single copy orthologs in our tested isolates. The number of single copy orthologs is larger than that (6976) in Chiapello et al. 2015 [29]. This discrepancy may be caused by differences in the method of gene prediction. After removing proteins encoded by genes that lacked start and/or stop codons and those with ambiguous orthologous relationships, 3257 single-copy orthologs were subsequently used in the phylogenetic analysis. To investigate the evolutionary relationships among $M$. oryzae and $M$. grisea, we generated a phylogenetic tree based on the third codon position of the single orthologous gene sets using a Maximum likelihood model (Fig. 1a). Since nucleotides in the third codon position are supposed to be under lower selection pressure than those in other codon positions, we expect the tree topology is more reflective of the evolutionary divergence among the isolates. The branch length corresponds to genetic distance based on nucleotide substitutions per site. This tree reconfirmed that $M$. grisea is genetically

Table 1 Summary of de novo assembly and gene prediction

\begin{tabular}{|c|c|c|c|c|c|c|c|c|}
\hline \multirow[b]{2}{*}{ Isolate } & \multirow[b]{2}{*}{ Ina168 } & \multirow[b]{2}{*}{$Y 34^{\mathrm{a}}$} & \multirow[b]{2}{*}{$\mathrm{P} 131^{\mathrm{a}}$} & \multicolumn{4}{|l|}{ Isolates } & \multirow[b]{2}{*}{ Dig41 } \\
\hline & & & & GFSI1-7-2 & $\mathrm{Br} 48$ & $\operatorname{Br} 58$ & Z2-1 & \\
\hline Number of corrected short reads (Million reads) & 57.6 & & & 60.7 & 56.6 & 53.5 & 61.0 & 60.1 \\
\hline Total size (Gbp) & 4.32 & & & 4.55 & 4.24 & 4.01 & 4.58 & 4.51 \\
\hline k-mer size & 37 & & & 38 & 39 & 35 & 41 & 40 \\
\hline Number of contigs ( $>=0.5 \mathrm{Kbp}$ ) & 1811 & & & 1910 & 2082 & 2057 & 2048 & 3970 \\
\hline N80 (Kbp) & 22.7 & & & 30.0 & 30.3 & 31.3 & 25.3 & 10.1 \\
\hline N50 (Kbp) & 77.1 & & & 88.3 & 97.5 & 91.1 & 64.1 & 24.3 \\
\hline Max contig length (Kbp) & 400.0 & & & 393.6 & 475.6 & 640.2 & 370.9 & 148.9 \\
\hline Total assembly size (Mbp) & 38.0 & & & 39.1 & 40.7 & 40.2 & 39.5 & 41.3 \\
\hline Total number of genes & 12,486 & 12,461 & 12,363 & 12,468 & 12,671 & 12,626 & 12,383 & 11,457 \\
\hline Number of completed genes & 11,910 & 12,106 & 11,897 & 11,866 & 12,020 & 12,009 & 11,925 & 9204 \\
\hline Number of genes with the start codon & 12,013 & 12,110 & 11,959 & 11,954 & 12,071 & 12,058 & 11,897 & 10,139 \\
\hline Number of predicted secretomes & 1747 & 1724 & 1723 & 1770 & 1768 & 1746 & 1793 & 1309 \\
\hline
\end{tabular}

aY34 and P131 data are from Xue et al. [18]

SignalP was performed for genes with the start codon. We did not perform SignalP for genes without the start codon 
a)

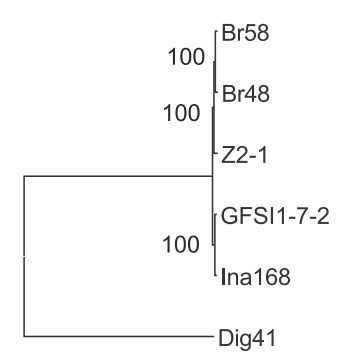

0.05

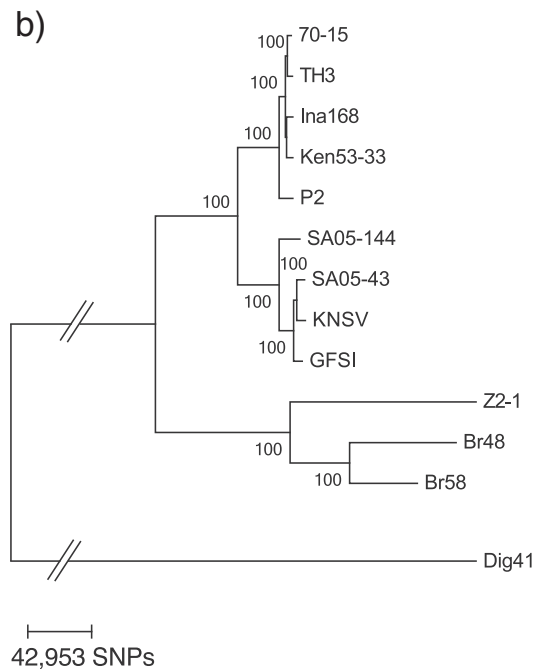

42,953 SNPS

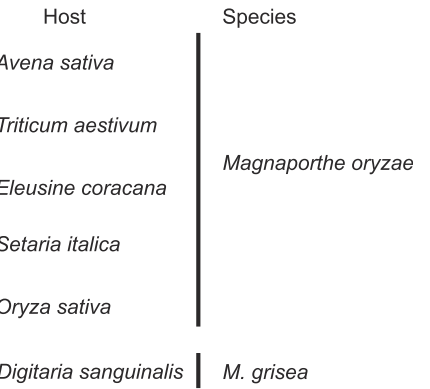

O. sativa

O. sativa

O. sativa

O. sativa

O. sativa

S. viridis

S. viridis

S. viridis

S. italica

E. coracana

T. aestivum

A. sativa

\begin{tabular}{l|l} 
D. sanguinalis & M. grisea
\end{tabular}

Fig. 1 Phylogenetic relationship for Magnaporthe oryzae infecting crops and M. grisea. a The maximum likelihood (ML) tree based on third codon positions of 3257 single copy genes. b The ML tree based on 859,067 SNPs distributed on the whole genome. M. grisea (Dig41) was used as an outgroup. The numbers on the branches indicate bootstrap probability. The right of the tree show their corresponding host species. The bar below the tree shows genetic distance per site

divergent from $M$. oryzae $[1,5,34,35]$. Furthermore, the M. oryzae isolates from Oryza and Setaria grouped within a single clade that was clearly separated from the clade containing M. oryzae isolates from Eleusine, Triticum and Avena. Avena isolate was closer to Triticum isolate than to Eleusine isolate. This result is consistent with the previous phylogenetic analyses $[1,5,29]$.

To confirm the observed phylogenetic relationship, we aligned the genomic reads from all $M$. oryzae isolates including additional isolates from Oryza and Setaria to the 70-15 reference genome [2], and constructed a phylogenetic tree based on all the single nucleotide polymorphisms (SNPs) that were estimated from the alignments. The breadth of coverage for the $11 M$. oryzae isolates ranged from 90.8 to $98.6 \%$ and the read depth ranged from 16 to 78 fold (Additional file 1: Table S3). Using these alignments, we identified a total of 859,067 sites that introduced a SNP in at least one isolate. These 859,067 SNP sites were used to construct a phylogenetic tree of the $11 M$. oryzae isolates and M. grisea Dig41 using a maximum likelihood (ML) model (Fig. 1b). The tree topology was consistent with that of the tree based on the third codon positions of single-copy orthologous genes (Fig. 1a), with the Oryza isolates clearly separated from the Setaria isolates.

Gene loss and gain predominantly occurred during host specialization in the Oryza-specific subgroup of $M$. oryzae For M. oryzae isolates from Oryza, gain and loss of avirulence effector genes is one of the major mechanisms to avoid effector-triggered immunity of host rice cultivars $[19,20]$. However, the contribution of gain and loss of genes in driving host specificity remains unclear. To evaluate this for five representative isolates of M. oryzae infecting Oryza, Setaria, Eleusine, Triticum, and Avena, we aligned the reads from each isolate to the genome assemblies of all other isolates to determine the breadth of coverage for each gene (Additional file 2). 
Genes with zero breadth of coverage were considered as absent in a particular pair-wise comparison [36]. Of all the genes, $1.2-3.5 \%(151-441)$ were identified as absent in different comparisons (Fig. 2a). Overall the loss of genes appears to have more frequently occurred in the $M$. oryzae lineages that infect Oryza and Setaria, with the same tendency reflected in analysis of the predicted secretome. Specially, the Oryza Ina168 isolate lost the largest number of genes (345-441) from the reference genomes of Setaria, Eleusine, Triticum and Avena isolates.

To further investigate the role of presence/absence polymorphisms in host specialization, we considered genes that were uniquely present or absent in each lineage of the host-specific subgroups of $M$. oryzae. We assigned loss and gain of genes into three categories: (1) genes that were lost once in the evolutionary history of $M$. oryzae, (2) genes that were subjected to multiple deletion events from different processes such as horizontal gene transfer and recombination, and (3) genes that uniquely existed in one host-specific subgroup. The number on each branch of the tree indicates the number of genes belonging to the first category (Fig. 2bc). The genes in the third category were treated as gained genes in each lineage of the host-specific subgroups. Remarkably, more deletion events occurred after the separation of isolates pathogenic on Oryza and Setaria (134-153) as compared to those of Triticum and Avena (8-18) although the genetic distance per synonymous site

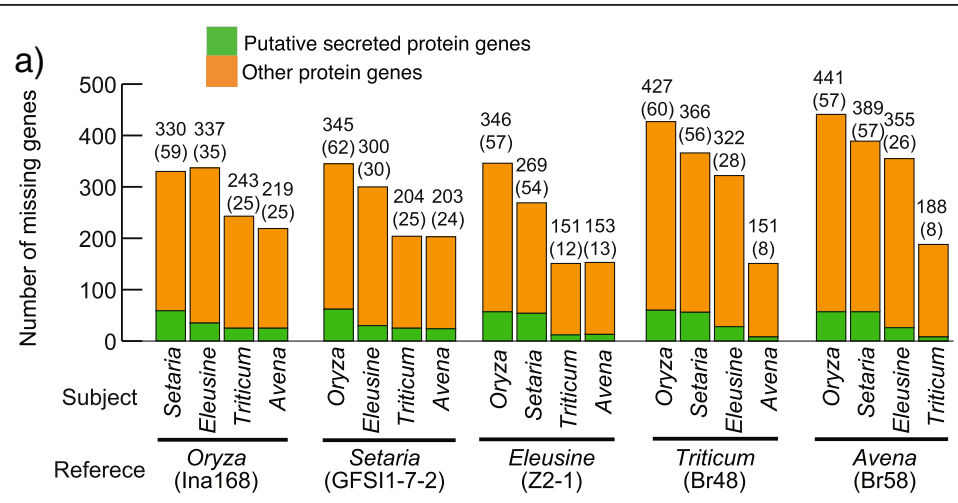

b)

Gene models Ina168 A GFSI1-7-2 Z2-1 Br48 $\triangle$ Br58

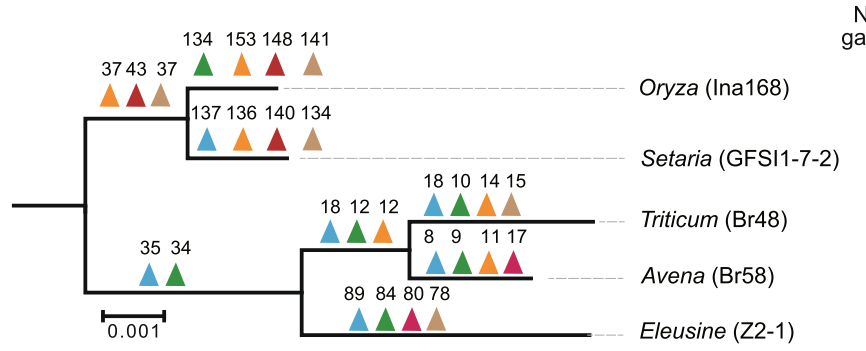

Number of gained genes

97

48
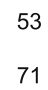

c)

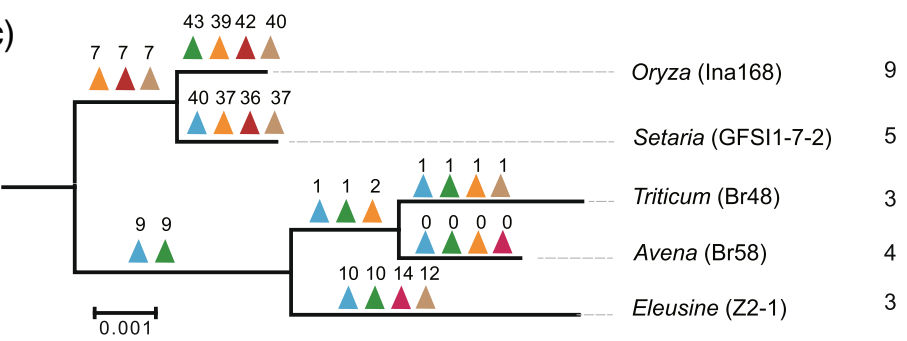

Fig. 2 The gain and loss of genes in the five representatives of Magnaporthe oryzae host-specific subgroups. a The bar plot shows the number of missing genes from each reference genome. The number of secreted protein genes is shown in parentheses. $\mathbf{b}$ The event of gain and loss of genes on the evolutionary history of the five representatives of $M$. oryzae. $\mathbf{c}$ The event of gain loss of putative secreted protein genes. The triangles indicate deletion events for each gene model of the host-specific subgroups of $M$. oryzae. The numbers above the triangles are corresponding to the number of genes that was lost once in the evolutionary history of $M$. oryzae. Blue, green yellow, red and ivory indicate the gene models of Oryza isolate Ina168, Setaria isolate GFSI1-7-2, Eleusine isolate Z2-1, Triticum isolate Br48, and Avena isolate Br58, respectively. At the right of the tree, the number of gain of genes for each host-specific subgroup was shown 
between the Oryza and Setaria isolates $(0.0027 \pm 0.0001)$ was smaller than that between the Triticum and Avena isolates $(0.0053 \pm 0.0000)$. This tendency was more prominent when considering genes encoding putative secreted proteins (Fig. 2c and Additional file 3: Figure S2); 36 to 43 genes were lost for isolates from Oryza and Setaria compared to 0 to 1 uniquely lost for isolates pathogenic on Triticum and Avena. In addition, M. oryzae isolates from Oryza gained the largest number of genes (Fig. 2bc, Additional file 3: Figure S1 and Figure S2), although the number of gained genes did not have such large difference than that of absent genes among the host-specific subgroups.

Loss of genes is independent of divergence between $M$. oryzae isolates unlike nonsynonymous nucleotide substitutions

Nucleotide substitutions can contribute to host specialization [37]. To determine the extent of nucleotide changes during host specialization of $M$. oryzae isolates, we estimated the ratio of nonsynonymous substitutions to synonymous substitutions $(\mathrm{dN} / \mathrm{dS})$, which is commonly used as a parameter of positive selection (Additional file 2). Genes encoding proteins contributing to host adaptation tend to show a dN/dS ratio over one [36, 37]. At first, we estimated thresholds of $\mathrm{dN} / \mathrm{dS}$ for detecting outliers in each comparison between the representative isolates when genes with $\mathrm{dN} / \mathrm{dS}=99(\mathrm{dS}=0$ and $\mathrm{dN}>0)$ were included (Additional file 1: Table S4) or excluded (Additional file 1: Table S5). Genes with outlier ratios of dN/dS are potential fast-evolving genes. As observed in $M$. oryzae effector AVR-Pik [21, 22], genes with $\mathrm{dN} / \mathrm{dS}=99$ may be involved in interaction with host molecules and subjected to positive selection. On the other hand, a gene with one nonsynonymous substitutions is likely to have experienced different selection pressures compared to a gene with a larger number of nonsynonymous substitutions, although both genes would show $\mathrm{dN} / \mathrm{dS}=99$ [38]. Given this issue, we reported results including/excluding genes with $\mathrm{dN} /$ $\mathrm{dS}=99$. Since thresholds of $d N / d S$ were less than one in most of pairwise comparisons between the representative isolates when genes with $\mathrm{dN} / \mathrm{dS}=99$ were excluded (Additional file 1: Table S5), $\mathrm{dN} / \mathrm{dS}=1.5$ was used as a threshold value. When genes with $\mathrm{dN} / \mathrm{dS}=99$ were included, 0-6.5 \% (0-805) of all the genes showed outlier values of $\mathrm{dN} / \mathrm{dS}$ in different comparisons (Fig. 3a). When genes with $\mathrm{dN} / \mathrm{dS}=99$ were excluded, genes with outlier values of $\mathrm{dN} / \mathrm{dS}$ added to $0.2-0.8 \%(26-94)$ of all the genes (Fig. 3b), a proportion that is much smaller than when genes with $\mathrm{dN} / \mathrm{dS}=99$ were included. However, both of the cases showed similar tendency in the proportion of genes with outlier values of $\mathrm{dN} / \mathrm{dS}$. When we compared the Oryza isolate Ina168 and Setaria isolate GFSI1-7-2 to Triticum (Br48) and Avena (Br58) isolates, a higher proportion of genes showed outliers of dN/dS. When the Triticum isolate $\mathrm{Br} 48$ and Avena isolate $\mathrm{Br} 58$ were compared to Oryza (Ina168) and Setaria (GFSI1-7-2) isolates, a higher proportion of genes showed outliers of $\mathrm{dN} / \mathrm{dS}$.

To examine how this compared to the presence/absence analysis, we plotted the number of genes with outlier values of $d N / d S$ when genes with $d N / d S=99$ were included or excluded or number of genes identified as absent in the reference genome against the genetic distance at synonymous sites $(\mathrm{dS})$, which is proportional to time from separation for the two pathogen isolates (Fig. 3c). The number of genes with outlier values of $\mathrm{dN} / \mathrm{dS}$ value was positively correlated with $\mathrm{dS}$, regardless of inclusion or exclusion of genes with $\mathrm{dN} / \mathrm{dS}=99$. However, the number of absent genes was not correlated with the $\mathrm{dS}$ values. This indicates that loss of genes was independent of divergence between $M$. oryzae isolates, but the accumulation of nonsynonymous substitutions was dependent on divergence time.

To identify genes that may have specifically evolved in each host-specific subgroup, we categorized genes that showed an outlier $\mathrm{dN} / \mathrm{dS}$ into 15 patterns (Additional file 3: Figure S3A). The patterns 1 to 4 represent that genes may have rapidly evolved in a specific pair of the representative isolates and were potentially involved in specialization in one isolate of the pair or both isolates. The Venn diagrams in Additional file 3: Figure S3 and Figure S4 were described based on these 16 patterns. A larger number of genes with outlier values of $\mathrm{dN} / \mathrm{dS}$ was shared between phylogenetically close subgroups. For instance, Oryza isolate Ina168 shared 119 genes with Setaria isolate GFSI1-7-2, but only 39 genes with Triticum isolate $\mathrm{Br} 48$ in the Z2-1 gene model (Additional file 3: Figure S3D). Genes encoding putative secreted proteins showed similar patterns (Additional file 3: Figure S3).

\section{Redundancy of functional domains in the genome promotes genetic variation in host-specific subgroups of M. oryzae}

Gene deletion or loss of protein function via mutation is deleterious for pathogens if the proteins are essential for development, infection, virulence and/or protection from abiotic and biotic stress. Therefore, the impact of loss of genes and nonsynonymous substitutions to fitness should be different between functionally redundant and non-redundant genes. To test whether this principle was true for the observed variation in the host-specific subgroups of $M$. oryzae, first, we classified functional domains for the predicted proteins using Hidden Markov Model (HMM) analysis against the Pfam database (Additional file 2) [39]. Then, we categorized functional domains into two groups where: (1) domains were detected only once in the genome or, (2) domains were detected more than once in the genome. As expected, $M$. 

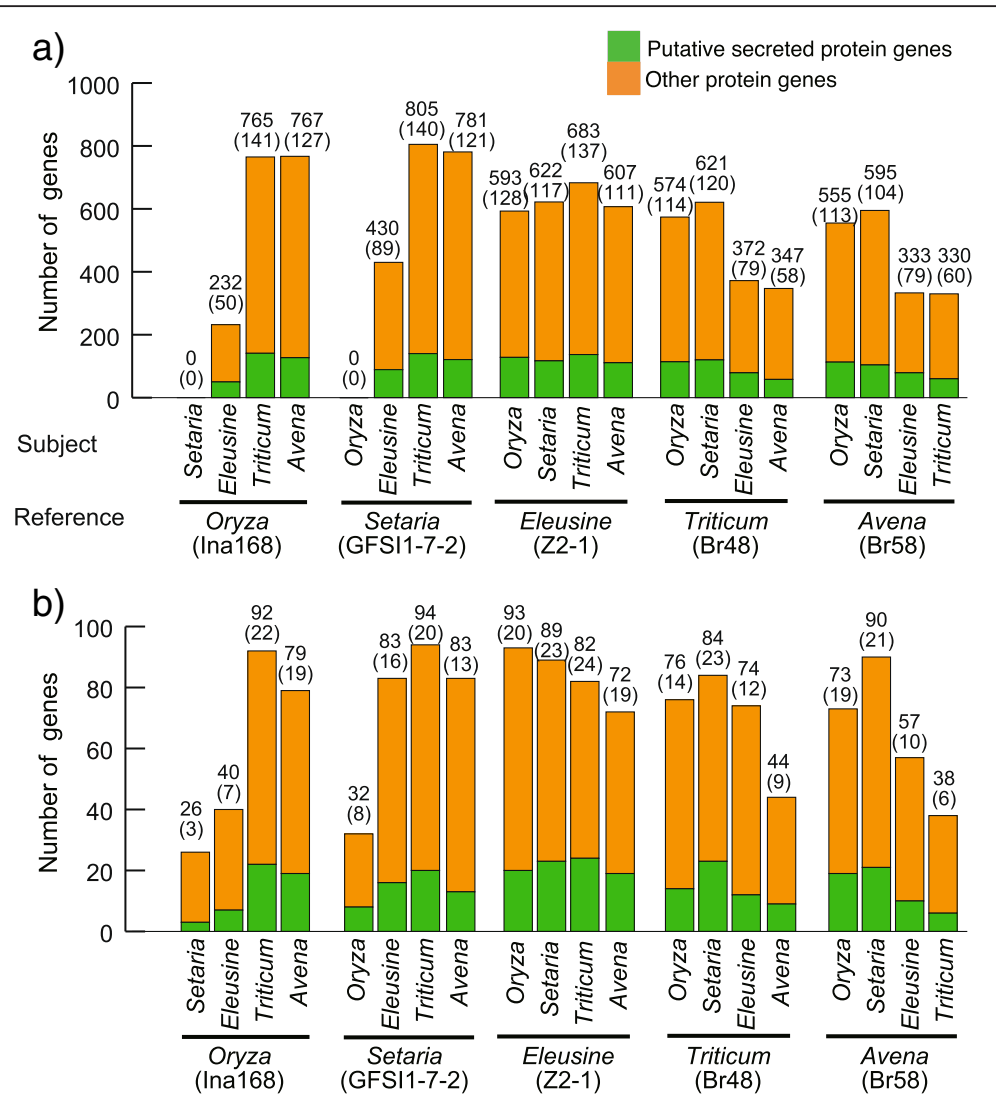

c)

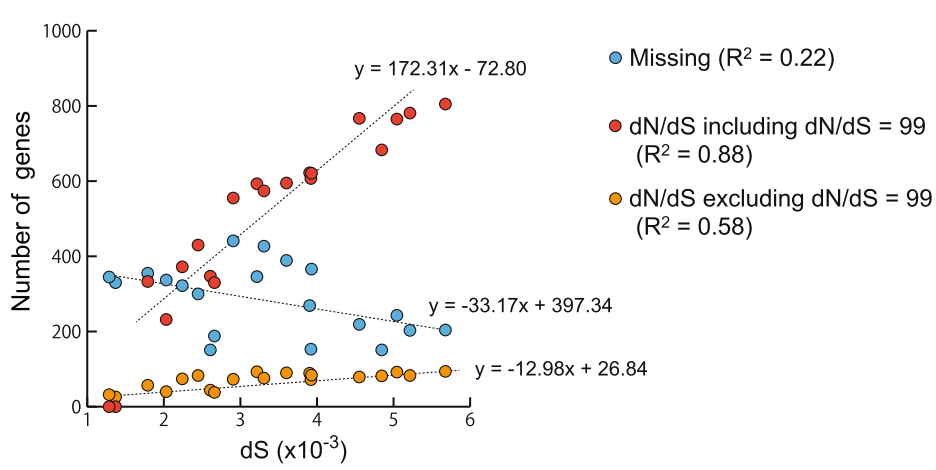

Fig. 3 A contrast pattern between nucleotide substitutions and gain/loss of genes in the host-specific subgroups of Magnaporthe oryzae. $\mathbf{a}-\mathbf{b}$ The bar plot shows the number of genes showing outlier values of $\mathrm{dN} / \mathrm{dS}$ between the reference isolate and each of other four representatives when $\mathrm{dN} / \mathrm{dS}=99 \mathrm{was}$ included (a) or excluded (b). The green and orange bars are corresponding to secreted protein genes and non-secreted protein genes, respectively. The number of secreted protein genes is shown in parentheses. $\mathbf{c}$ The plot between the number of polymorphic genes and the number of synonymous substitutions per site. The red and blue circles indicate missing genes and genes showing $\mathrm{dN} / \mathrm{dS}>1.5$, respectively. Each of the trend lines is shown

oryzae preferentially lost genes (Fig. 4a) or gained nonsynonymous mutations in genes whose domains are present in multiple copies in the genome (Fig. $4 \mathrm{~b}$ and c).

To further investigate genes that may be involved in host adaptation, we examined the functional domains identified in proteins that were encoded by genes that were subject to isolate specific loss and/or had an outlier value of $\mathrm{dN} / \mathrm{dS}$ in one specific pair of the representative isolates. Only a small number of domains (0-13.9 \%) were shared between proteins independently lost for each lineage in the host-specific subgroups (Fig. 4d). Similar results were obtained when considering proteins encoded by genes with outlier values of $\mathrm{dN} / \mathrm{dS}$ regardless of inclusion (Fig. 4e) or exclusion of genes with $\mathrm{dN} / \mathrm{dS}=99$ (Fig. 4f). These results indicate that deletions and nonsynonymous substitutions occurred in genes encoding functionally different proteins after the separation from each of their common ancestors. We could not find statistical enrichment of specific functional domains in the proteins uniquely lost and evolving for each pathogen subgroup. 
a)

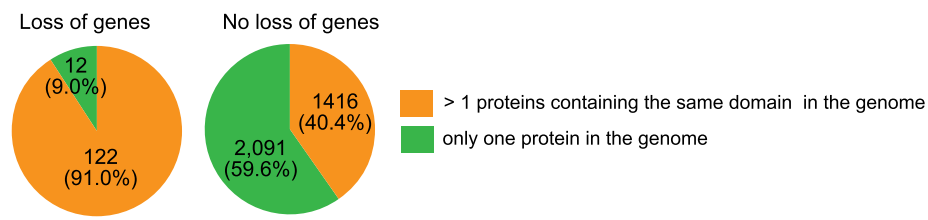

b) $d N / d S=99$ was included

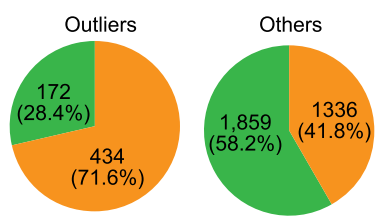

C) $\mathrm{dN} / \mathrm{dS}=99$ was excluded.

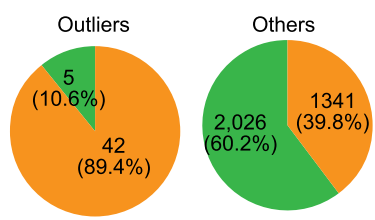

d) Gain/loss of genes
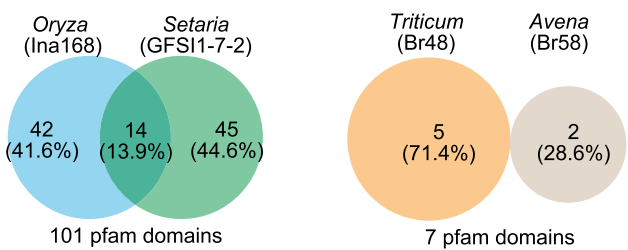

e) dN/dS
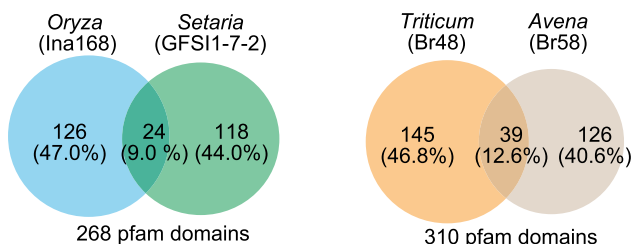

f) $\mathrm{dN} / \mathrm{dS}$ (dN/dS = 99 was excluded.)
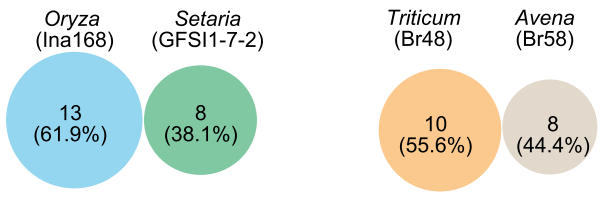

21 pfam domains

18 pfam domains

Fig. 4 Characterization of genes showing gain/loss and excess of nonsynonymous substitutions. a-c The pie charts indicate the number of proteins whose domains were detected more than once or only once in the genome in four categories. The high percentage of genes experiencing loss (a) and with outlier values of $\mathrm{dN} / \mathrm{dS}$ when $\mathrm{dN} / \mathrm{dS}=99$ was included (b) or excluded (c) encodes proteins whose domains are present in multiple copies in each genome. $\mathbf{d}$ The percentage of shared domains of proteins whose genes showing presence/absence polymorphisms between two host-specific subgroups. e-f The percentage of shared domains of proteins whose genes showing outlier values of dN/dS between two host-specific subgroups when $\mathrm{dN} / \mathrm{dS}=99$ was included (e) or excluded (f). The numbers in (d-f) are corresponding to the number of pfam domain. The values in (a-f) are shown when Z-1 gene model was used

\section{Highly polymorphic effectors identified between and within the host-specific subgroups of $M$. oryzae}

To test how many genes encoding proteins shown to be involved in various stages of appressorium development and infection showed loss among host-specific subgroups of $M$. oryzae, which are absent from at least one of the subgroups, we sorted genes based on the 70-15 strain published gene models [2] and gene categorization [40] (Fig. 5a). As expected from previous studies [18, 19], effector genes showed most frequent loss (52.9 \%). In contrast, secreted glycosyl hydrolase, carbohydrate esterases and polysaccharide lyases were not frequently lost (5.7\%), which is consistent with the observation in Chiapello et al. 2015 [29]. Interestingly, 20 \% of genes encoding enzymes that utilize malonyl-CoA and key secondary metabolic enzymes showed presence/absence polymorphisms, contrasting with other enzymes that were monomorphic. This result is different from the conclusion based on clustering of orthologous genes with OrthoMCL [33], in which secondary metabolism genes were highly conserved. OrthoMCL used protein sequences, while our study estimated presence/absence polymorphisms based on the breadth of coverage of DNA sequence reads over genes. Being more sensitive to 
a)

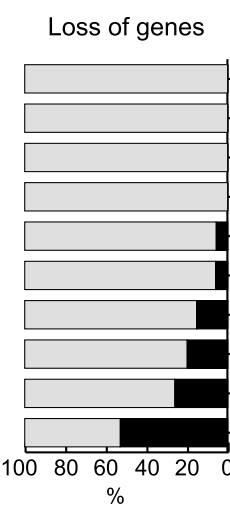

Hydrophobins and HsbA like-proteins
Enzymes involved quinate utilisation and shikimate pathway Enzymes involved in fatty acid
oxidation and glyoxylate cycle Enzymes that directly utilise or produce acetyl-CoA Secreted glycosyl hydolases, carbohydrate esterases and polysaccharide lyases Sugar transporters

Drug transporters

Enzymes that utilise malonyl-CoA Key secondary metabolic enzymes Effector

Outliers $\mathrm{dN} / \mathrm{dS}$

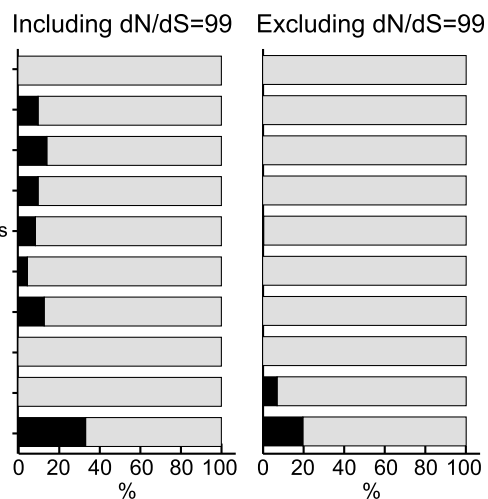

b)

Effector genes

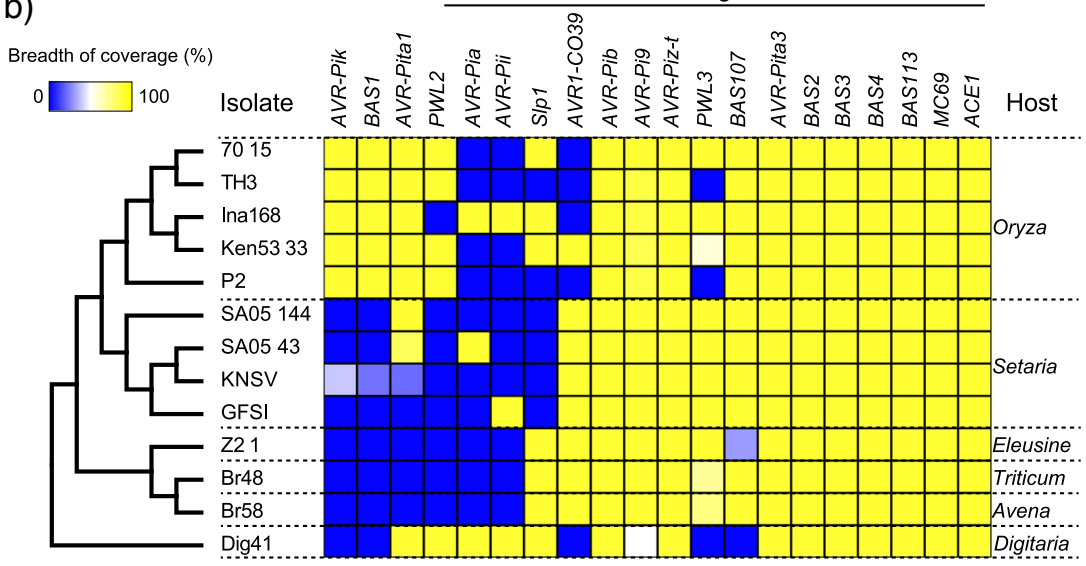

Fig. 5 Highly polymorphic in the effector genes between and within the host-specific subgroups of $M$. oryzae. a The percentage of genes showing loss, outlier values of dN/dS when dN/dS was included or excluded for each functional category. The black and gray bars indicate the percentage of genes showing loss or outlier values of dN/dS and the others, respectively. These percentages were calculated when we used $70-15$ strain genome as the reference. $\mathbf{b}$ Distribution of presence and absence of genes encoding the known effectors in $M$. oryzae and $M$. grisea. Heat map shows breadth coverage of genes. The blue and yellow panels indicate absence and presence polymorphisms, respectively. The tree indicates the relationship among the tested pathogens based on Fig. 1b

nucleotide differences, our method tends to detect more presence/absence polymorphisms. We also investigated genes showing $\mathrm{dN} / \mathrm{dS}$ between the 70-15 strain and each isolate of the host-specific subgroups. Except for genes encoding Hydrophobins and Hydrophobic surface binding protein A (HsbA) like-proteins, Enzymes that utilise malonyl-CoA, and Key secondary metabolic enzymes, all the tested categories included genes with an outlier value of $\mathrm{dN} / \mathrm{dS}$ when genes with $\mathrm{dN} / \mathrm{dS}=99$ were included (Fig. 5a). Furthermore, $33.3 \%$ of genes encoding effectors showed outlier values of $\mathrm{dN} / \mathrm{dS}$. When genes with $\mathrm{dN} / \mathrm{dS}=99$ were excluded, genes with outlier values of $\mathrm{dN} / \mathrm{dS}$ were detected only in effectors and key secondary metabolic enzymes.

We also specifically tested presence/absence of previously identified effector genes [41] AVR-Pia, AVR-Pii, AVR-Pik [19], PWL2 [42], PWL3 [43] AVR-Pita1 [44],
AVR-Pita3 [45], AVR-Piz-t [46], AVR1-CO39 [47], BAS14 [48], BAS107, BAS113 [49], AVR-Pi9 [50], AVR-Pib [51], S1p1 [52], MC69 [53], ACE1 [54] in M. oryzae representative isolates together with the $M$. grisea Dig41 isolate (Fig. 5b). In addition, presence/absence of the effector genes in three Oryza isolates and three Setaria isolates, which were re-sequenced for the analysis of genetic polymorphisms within these isolates, was also tested. The effector genes in $M$. oryzae were highly polymorphic in isolates infecting Oryza and Setaria. AVR-Pita1, AVR-Pia and AVR-Pii were missing in isolates infecting other cereals, but were present in $M$. grisea isolate Dig41. Interestingly, S1p1, an effector containing a LysM domain [52] was lost in all tested Setaria isolates and was polymorphic in the Oryza isolates, suggesting that Slp1 might be associated with host specialization. 
Lower nucleotide diversity but more frequent nonsynonymous mutations for $M$. oryzae isolates pathogenic on Oryza spp

Although Oryza and Setaria-specific subgroups are not separate species, the estimation of intra-specific variation is useful to test how different host environments have shaped genetic variations in $M$. oryzae. The representative isolate and the three additional isolates of each subgroup were used for the analyses of intra-specific variation. At first, to address gain and loss of genes involved in host specialization in the Oryza and Setariaspecific subgroups, we identified presence and absence polymorphisms in each population of $M$. oryzae on Oryza and Setaria (Additional file 3: Figure S5). The number of presence/absence polymorphisms in the $M$. oryzae Oryza subgroup was larger than that for the Setaria subgroup. This tendency was also more pronounced for genes encoding predicted secreted proteins. These observations were detected in all the gene models estimated from the genome sequences of 70-15 and the representative isolates.

We also calculated nucleotide diversity $(\pi)$ in $M$. oryzae Oryza and Setaria-specific subgroups (Table 2). The isolates pathogenic on Oryza spp. contained about half the nucleotide diversity of the isolates pathogenic on Setaria spp. for both coding and non-coding regions.

To further assess the process of DNA polymorphism accumulation between these populations, we applied the McDonald and Kreitman's (MK) test [55] to the observed polymorphisms in the gene coding regions (Table 3). In the neutral hypothesis of molecular evolution, the ratio of synonymous to nonsynonymous divergences between the populations would be expected to be similar to the ratio of synonymous to nonsynonymous polymorphisms within each population. When the MK test was applied to each gene, none of the tests gave significant results following multiple corrections. On the other hand, when DNA variation in all the tested genes were combined, for both M. oryzae Oryza and Setaria subgroups, the synonymous/nonsynonymous ratio of DNA polymorphisms levels did not conform to that of divergence (Table 3). In particular, it is notable that there are more SNPs causing nonsynonymous changes (4408) than those causing synonymous changes (3312) within the isolate infecting Oryza spp.

Table 2 Summary of nucleotide diversity (л) of Oryza and Setaria isolates

\begin{tabular}{llll}
\hline Group & \multicolumn{2}{l}{ Gene coding region } & Non-coding region \\
\cline { 2 - 3 } & \multicolumn{2}{l}{ Synonymous } & Nonsynonymous \\
\hline $\begin{array}{l}\text { Oryza pathogen } \\
(n=5)\end{array}$ & 0.00044 & 0.00019 & 0.00035 \\
$\begin{array}{l}\text { Sataria pathogen } \\
(n=4)\end{array}$ & 0.00101 & 0.00031 & 0.00073 \\
\hline
\end{tabular}

Tight linkage between transposable elements and polymorphic genes in $M$. oryzae isolates infecting Oryza spp. and Setaria spp

In $M$. oryzae, effector genes displaying presence/absence polymorphisms are often located next to transposable elements (TEs) $[15,18,19]$. For example, Slp1 of Oryza isolate Ina168 resides between two multiple mapping read-rich regions including partial sequences of long terminal repeat (LTR) retrotransposons (Fig. 6a). It is interesting to note that Slp1 is missing from Setaria isolate GFSI1-7-2 but the flanking multiple mapping read-rich regions are conserved (Fig. 6a), suggesting possible excision of Slp1 in Setaria isolates. Furthermore, TEs are known to contribute to insertion and deletion of DNA segments within the genome and also contribute to duplication of certain regions. To further investigate the relationships between transposable elements and the presence/absence of linked genes, we classified $M$. oryzae genes according to their distance to the closest TEs. Since the distribution of distances between the gene and TE were highly skewed for both 5'- and 3'-directions (Additional file 3: Figure S6), we decided to classify them non-parametrically to the first quartile $(\mathrm{C} 1$ : genes close to $\mathrm{TE},<25 \%$ of the total genes), the last quartile (C3: genes most distant from $\mathrm{TE},<25 \%$ of the total genes) and in between (C2: 25-75 \%). We observed that genes tightly linked to TEs showed a higher incidence of presence/absence polymorphisms within Oryza and Setaria isolates (Fig. 6bc).

Next, to test whether TEs influence the level of nucleotide substitutions and polymorphisms, we separately calculated nucleotide diversity ( $\pi$ ) for $M$. oryzae Oryza and Setaria specific subgroups, respectively, genetic distance $(\mathrm{Da})$ between Oryza and Setaria specific subgroups for Supercontigs 8.1 to 8.7 (Fig. 7a). The values of Oryza $\pi$, Setaria $\pi$ and $D a$ were variable along the selected genomic regions for all contigs. There was an obvious correlation among these three values (Fig. 7a, Additional file 3: Figure S7). We also addressed the position of transposable elements (TEs) on the genome and studied the relationship between TE location and values of Oryza $\pi$, Setaria $\pi$ and $D a$. As seen in Fig. 7a, there was a tendency for genomic regions with a higher density of TE's to show higher values of Oryza $\pi$, Setaria $\pi$ and $D a$ (Fig. 7bcd). TE density had a statistically significant positive correlation with Oryza $\pi$, Setaria $\pi$ and Da. Note that values of Oryza $\pi$, Setaria $\pi$ and $D a$ were calculated after removing the DNA sequences corresponding to transposable elements. These results suggest that genomic regions close to transposable elements tend to harbor higher levels of nucleotide diversity within populations and larger divergence between populations.

To further investigate the relationships between the transposable elements and DNA polymorphisms of the 
Table 3 Results of McDonald and Kreitman's (MK) test applied to Oryza and Setaria isolates of M. oryzae

\begin{tabular}{|c|c|c|c|c|}
\hline & Synonymous & Nonsynonymous & $x^{2}$ & $P$ \\
\hline Fixed differences between Oryza isolates and Setaria isolates & 7631 & 4940 & & \\
\hline Polymorphisms in Oryza isolates & 3312 & 4408 & 609.32 & $P<2.2 \mathrm{e}-16$ \\
\hline Polymorphisms in Setaria isolates & 5933 & 5411 & 171.15 & $P<2.2 \mathrm{e}-16$ \\
\hline
\end{tabular}

linked genes, we classified M. oryzae genes according to their distance to the closest TE according to the same criteria of the analysis of presence/absence polymorphisms (Fig. 6c). For each class of C1, C2 and C3, the level of presence/absence polymorphisms, nucleotide diversity for synonymous and nonsynonymous DNA polymorphisms and divergence between Oryza and Setaria pathogens (Da) was examined (Additional file 3: Figure S8). We noted that genes closely linked to TEs (C1) showed significantly higher levels of presence/absence polymorphisms
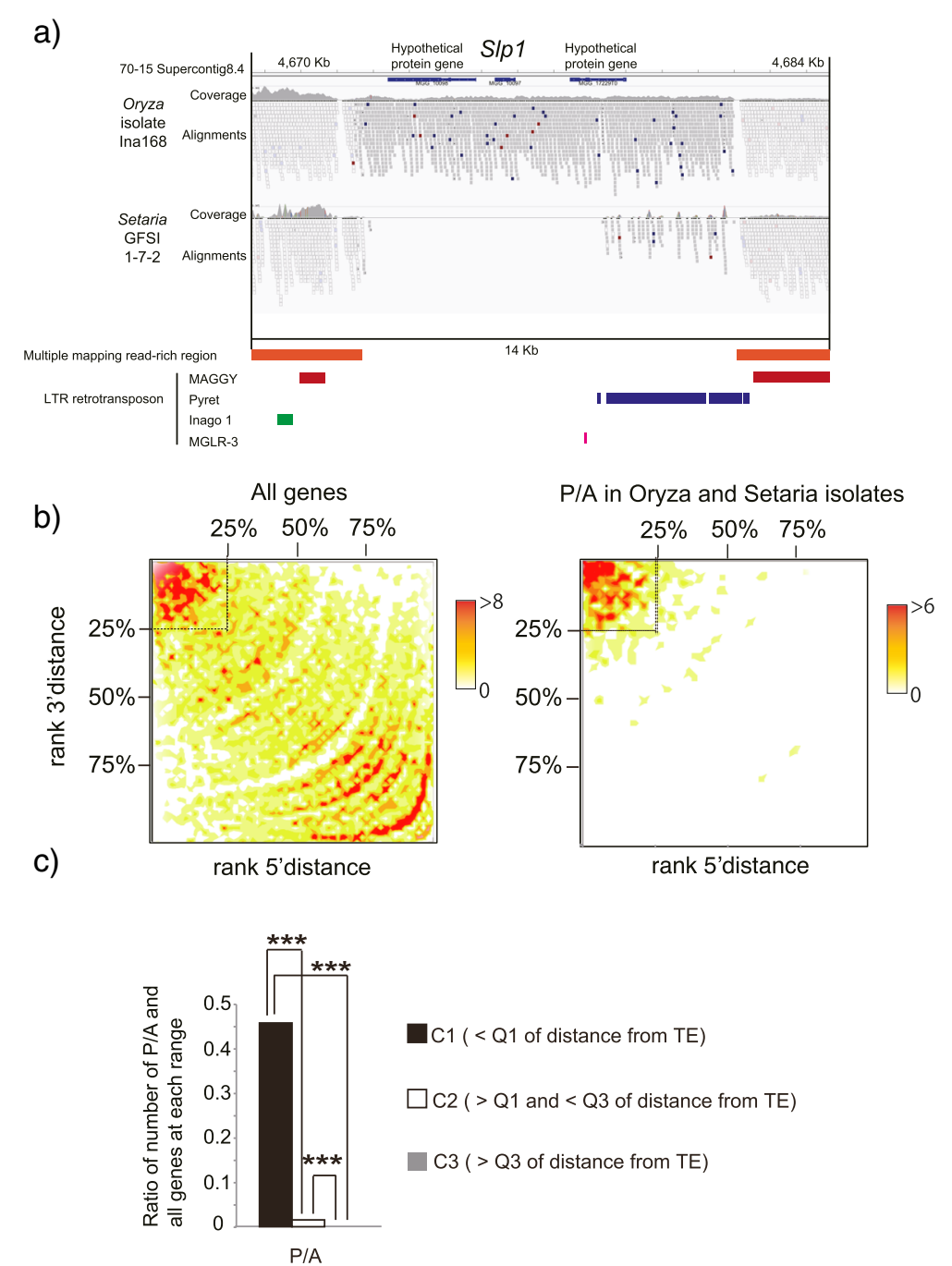

Fig. 6 Genes showing presence and absence polymorphisms are linked to transposable elements. a The alignments view of short reads around SLP1 region. SLP1 was surrounded by multiple mapping reads and partial LTR retrotransposons. b Distribution of presence and absence polymorphisms within Oryza and Setaria isolates according to local TE density. Magnaporthe genes were ranked on the basis of ascending distance from 5'-end (y-axis) and $3^{\prime}$-end (x-axis) of genes to TEs. The number of genes corresponding to genes in each bin is shown as a heat map. 25, 50 and $75 \%$ indicate first quartile, median and third quartile, respectively. The left and right panels are the distributions of all genes and genes showing presence/ absence polymorphisms, respectively. c The bar plot shows the ratio of the number of genes showing presence and absence polymorphisms within Oryza and Setaria isolates to genes at each of category C1, C2, and C3. Statistical significance was evaluated by using exact Wilcoxon Mann-Whitney rank sum test (**: $P<0.001)$. Since the ratio in the $\mathrm{C} 3$ was very small, the value is not seen in the figure 


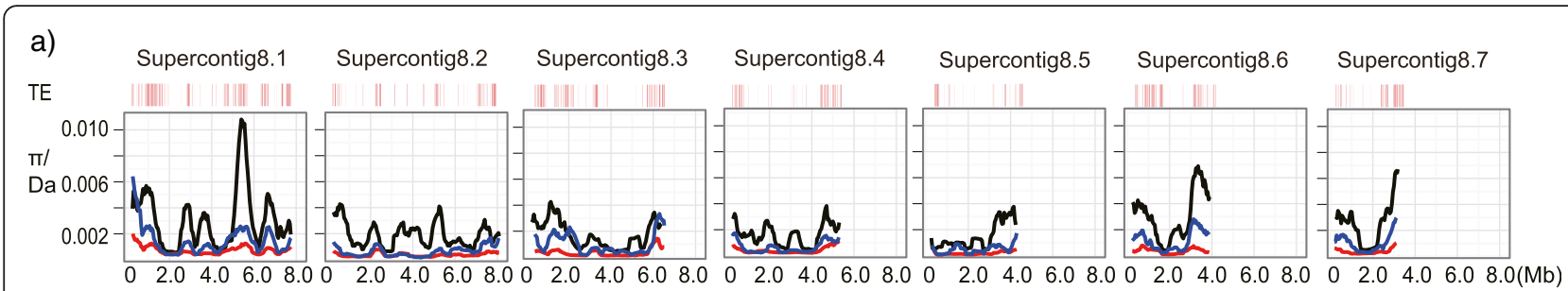

b)

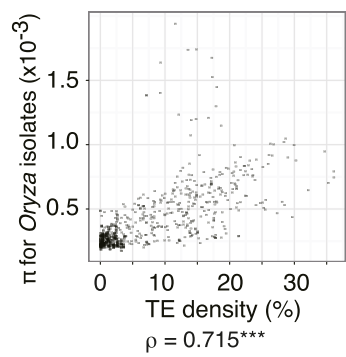

c)

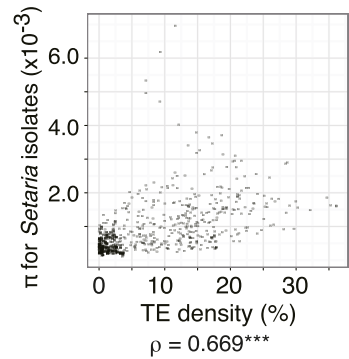

d)

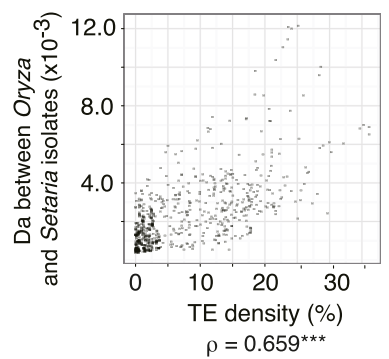

Fig. 7 The level of DNA polymorphisms in M. oryzae is correlated with the density of transposable elements. a Results of sliding window analysis of $\pi$ for Oryza isolates (red), $\pi$ for Setaria isolates (blue), and the number of net nucleotide substitutions per site (Da) (Nei 1987) between Oryza and Setaria isolates are given for seven supercontigs. These values were estimated based on nucleotide variations at synonymous sites and noncoding regions. Window size is $500 \mathrm{Kbp}$. Step size is $50 \mathrm{Kbp}$. TE: location of transposable elements. Correlation between the density of transposable elements and three measurements: nucleotide diversity $(\pi)$ of Oryza (b) and Setaria (c) isolates, and distance (Da) between Oryza and Setaria isolates (d)

and synonymous and nonsynonymous nucleotide polymorphisms and divergence between Oryza and Setaria pathogens than the genes distantly located to TEs ( $C 2$ and C3). From these results, we conclude that presence of transposable elements may increase genetic diversity and divergence of genes located nearby.

\section{Discussion}

Higher rates of gene loss in $M$. oryzae isolates from Oryza, Setaria, and Eleusine than in isolates from Triticum and Avena

The majority of genes were conserved across all tested host-specific subgroups of $M$. oryzae with only a small fraction (1.2-3.5 \% of the genes) dynamically gained and lost, as reported in Chiapello et al. 2015 [29]. Previous studies have suggested that the insertion of transposons and the positioning of genes close to telomeres may enhance mobility of genes via chromosomal rearrangements including translocation and deletion [12, 15, 44, 56]. In addition, our study revealed that functional redundancy may contribute to the dynamics of $M$. oryzae genes. Interestingly, we observed that in comparisons between host-specific subgroups, the isolate infecting rice showed the largest number of genes lost from the genome (345-441 genes), followed by the isolate from Setaria spp. (269-389 genes) and the isolate from Eleusine spp. (300-355 genes), respectively. The number of genes lost from the genomes of Triticum and Avena pathogens when compared to all other host-specific subgroups was relatively small (ranging from 151 to 243)
(Fig. 2a). These observations could be explained by the difference in evolutionary time following the host shift (divergence time). Accordingly, Oryza, Setaria, and Eleusine specific subgroups of $M$. oryzae are predicted to have a longer evolutionary history of host specificity when compared to Triticum and Avena specific subgroups. The origin of Oryza M. oryzae isolates was dated back to rice cultivation $\sim 10,000$ years ago [1], whereas Triticum isolates were first reported in the 1980s [23], suggesting a recent host jump and specialization. Following host shift and during the ensuing specialization period, deletions in non-essential and/or deleterious genes may have been retained through purifying selection or random genetic drift. Our analysis showed that $M$. oryzae preferentially lost genes whose domains were present as multiple copies in the genome and encoded different functional domains between isolates pathogenic on Oryza spp. and Setaria spp. In addition, selection pressures may vary between hosts and could also shape the distinct patterns between the host-specific subgroups. Intensive breeding of rice cultivars resistant to blast disease may have been more extensive compared with Setaria spp. and Eleusine spp. resulting in more frequent gain and loss of genes in M. oryzae isolates pathogenic on Oryza spp.

\section{Accumulation of non-synonymous substitutions in the host-specific subgroups of $M$. oryzae}

An excess of nonsynonymous mutations compared to synonymous mutations was found in $0-6.5 \%$ of genes in 
M. oryzae when genes with $\mathrm{dN} / \mathrm{dS}=99$ were included. Genes with outlier values of $\mathrm{dN} / \mathrm{dS}$ could be classified into two groups. One group was potentially under positive selection. Positive selection was common among the effector genes AVR-Pizt, Slp1, PWL3, AVR-Pita3 and $B A S 2$ (Fig. 5, Additional file 2), some of which were previously reported within Oryza isolates [20]. Since the avirulence effectors are considered to have coevolved with their cognate resistance genes of the host plants [57], an arms race with the host plant immune system could explain the excess of nonsynonymous substitutions (e.g. [21, 22]). Of the putative secreted protein genes, $0-1.1 \%$ showed outlier values of $\mathrm{dN} / \mathrm{dS}$ as observed in these avirulence proteins when genes with $\mathrm{dN} /$ $\mathrm{dS}=99$ were included. These proteins are presumably secreted outside the pathogen and possibly interact with host molecules, contributing to specialization to the host environment as observed in several pathosystems [17, 37].

Another group of genes with outlier values of $\mathrm{dN} / \mathrm{dS}$ had a few nonsynonymous substitutions and zero synonymous substitutions. Small synonymous divergence is not enough to give statistical power to detect positive selection [38]. The number of genes with outlier values of $\mathrm{dN} / \mathrm{dS}$ was positively correlated with genetic divergence between host specific subgroups. This observation is consistent with an overall random accumulation of nonsynonymous substitutions in this group and the degree to which these genes are under positive selection cannot be determined with the current data. As detected in the presence/absence polymorphism analysis, these genes generally encode proteins whose domains were present in multiple copies in the genome. Therefore, the observed nonsynonymous substitutions may not have had deleterious effects on the fitness of the pathogen due to the functional redundancy of the proteins targeted for nonsynonymous mutation. It is interesting to note, however, that some avirulence effectors that have no synonymous substitutions have nonsynonymous substitutions that impact their activity and thus in these cases the mutations are probably beneficial and positively selected (e.g. AVRPik/Pik, [21, 22]). The degree to which this affects the other genes in this category cannot be determined.

Recently, de Guillen et al. 2015 [58] reported the existence of a family of effectors (MAX-effectors) that has no nucleotide sequence homology but has conserved $3 \mathrm{D}$ protein structure. The protein structure of these effector proteins may enable the accumulation of nonsynonymous substitutions involved in the adaptation to various host species. The expansion and diversification of the MAX-effectors that was noted in M. oryzae [58] is consistent with our view that gene gain and loss are major drive of evolution in this species. In combination with the protein structure scaffold of the MAX-effectors, relaxation of purifying selection due to functional redundancy may have accelerated protein evolution in M. oryzae.

\section{The LysM domain containing effector SIp1 was lost in the} $M$. oryzae subgroup that specifically infects Setaria plants The effector BAS1 and $A V R-P i k$ are observed in the Oryza-specific subgroup and not in the Setaria-specific subgroup. These effectors have been shown to be specifically induced during the biotrophic phase of $M$. oryzae infection on rice $[48,59]$. This suggests they may contribute towards specialization of this $M$. oryzae subgroup to rice plants. On the other hand, the LysM domain effector gene Slp1 was missing in two Oryza isolates (TH3 and P2) and in the Setaria subgroup. The Slp1 is an apoplastic effector that binds Chitin and contributes to the avoidance of pathogen-associated molecular pattern (PAMP) triggered immunity [52]. Furthermore, knocking out of Slp1 reduced pathogenicity of M. oryzae on rice plants [52]. The 70-15 strain had eight putative secreted proteins containing LysM domains and the Oryza isolate Ina168 had one additional putative secreted protein containing a LysM domain. Furthermore, the Setaria-specific subgroup lost Slp1 but harbor an extra LysM domaincontaining protein, which potentially could compensate for the lack of Slp1. The loss of Slp1 from the Setariaspecific subgroup could be due to (1) functional redundancy driven by genetic drift, or (2) Slp1 being deleterious for adapting Setaria plants driving loss by purifying selection. As all other crop-infecting M. oryzae and also M. grisea Dig41 maintain Slp1, this suggests an essential role for the effector, with its loss in Setaria-specific subgroup likely a result of host specialization. However, we only examined a limited number of Setaria isolates. To challenge this hypothesis, it is necessary to survey a larger number of Setaria isolates to estimate the effector frequency in population of Setaria isolates.

\section{Linkage to transposons is associated with elevated mutation rates}

TEs are known to contribute to insertion, deletion and duplication of DNA segments in a genome. We observed that genes tightly linked to TEs show a higher incidence of presence/absence polymorphisms (Fig. 6). In addition to this effect, we also observed a higher level of nucleotide polymorphisms in the genes more closely linked to TEs than those distantly linked to TEs (Additional file 3: Figure S8). This observation suggests that TEs can generate higher nucleotide level diversity as well as structural diversity in genomes. In a species like $M$. oryzae that predominantly propagates asexually, genetic recombination by sexual crosses is limited. Asexual propagation limits genetic diversity that is needed for adaptation to new environments and enhances the accumulation of deleterious mutations (Muller's ratchet) reducing the 
mean fitness of the species (population). We hypothesize that TEs may substitute the function of sexual recombination to contribute to maintaining genetic diversity. For instance, genes closer to TEs could potentially be inadvertently mutagenized during imperfect TE-insertion mediated mismatch repair, thereby enhancing genetic diversity during prolonged periods of exclusively asexual reproduction.

\section{Abrupt changes in environment drove accumulation of nonsynonymous mutations and presence/absence polymorphisms in $M$. oryzae Oryza isolates}

We identified an excess of nonsynonymous DNA polymorphisms as compared to synonymous ones and presence/absence polymorphisms in the Oryza-specific subgroup. Since there is no wild rice species in Japan, Japanese Oryza isolates survive only on cultivated rice. In contrast, Setaria isolates can survive on wild Setaria species, which are commonly distributed across Japan. Therefore, Oryza M. oryzae isolates, are more vulnerable to artificial selection of rice breeding. These different selection pressures could generate more frequent presence/ absence polymorphisms in the Oryza-specific subgroup even though the level of nucleotide diversity in Oryza isolates was smaller than that in Setaria isolates. This excess of nonsynonymous polymorphisms in the Orzya subgroup could be explained by two general mechanisms. One is positive selection on protein coding genes in the Oryzaspecific subgroup. For example, AVR-Pik seems to be engaged in an arms race with its cognate resistance gene $P i k$ of rice $[21,22]$. The observed amino acid differences between AVR-Pik isoforms determine binding and activation of corresponding Pik immune receptor isoforms. The other is that this higher level of nonsynonymous polymorphisms in the isolates infecting Oryza spp. may be caused by relaxation of purifying selection by its smaller population effective size. In either mechanism, the relatively higher level of nonsynonymous DNA polymorphisms in the Oryza-specific subgroup may provide the genetic and phenotypic diversity that becomes favorable when the population encounters abrupt changes of environment, e.g. changes in host cultivars. Such high level of intraspecific genetic plasticity should facilitate the persistence of this pathogen lineage despite the constantly changing host environment.

\section{Conclusions}

This comparative genomics study sheds light on the evolutionary mechanisms underpinning $M$. oryzae specialization to host plant species. We investigated gain and loss of genes and nucleotide substitutions between host-specific subgroups of $M$. oryzae. We found that redundancy of functional domains in the genome may have enabled a high degree of intra-specific gain and loss of genes and accumulation of nonsynonymous substitutions. Interestingly, these two mechanisms of genetic variations showed contrasting patterns in the comparisons of the hostspecific subgroups (Oryza-Setaria vs. Avena-Triticum pathogens). The number of presence/absence of genes was not correlated with genetic divergence between the host specific subgroups, while the number of genes with outlier values of $\mathrm{dN} / \mathrm{dS}$ correlated well with genetic distance. Though the genetic distance between the isolates infecting Oryza and Setaria was smaller than that between those infecting Triticum and Avena, the loss and gain of genes in Oryza and Setaria isolates were more frequently observed than those in Triticum and Avena isolates. Considering that Oryza and Setaria specific subgroups of M. oryzae have a longer evolutionary history of host specificity when compared to Triticum specific subgroup, this observation suggests that the differences are not explained by the elapsed time of genetic isolation but rather by coevolution with the specific host. In addition, the study of variations in Oryza- and Setaria-specific subgroups of M. oryzae revealed that the isolates pathogenic on Oryza had higher number of nonsynonymous polymorphisms, despite having smaller nucleotide diversity than the isolates pathogenic on Setaria. Differences in hosts and frequency in abrupt changes of environments may have shaped the contrasting patterns of genetic variations between these hostspecific subgroups of M. oryzae.

\section{Methods}

\section{DNA extraction and sequencing}

We extracted DNA from mycelia of $M$. oryzae and $M$. grisea, which are maintained in Laboratory of Plant pathology, Kobe University, Iwate Biotechnology Research Center and National Institute of Agrobiological Sciences Genbank, using the DNeasy Plant Maxi kit (QIAGEN). Five $\mu \mathrm{g}$ of the DNA was used for constructing the paired-end libraries following manufacturer's instructions. The libraries were used for cluster generation on a flow cell and sequenced for 76 cycles on an Illumina Genome Analyzer IIx. Base calling and filtering of low quality bases were performed using sequence control software (SCS) real-time analysis, BCL converter and the GERALD module (Illumina, San Diego, CA, USA).

\section{De novo assembly of short reads and gene predictions}

Adapter sequences were removed from short reads adapter using a software cutadapt [60]. Unpaired reads were filtered using a perl program cmpfastq (http:// compbio.brc.iop.kcl.ac.uk/software/index.php). We conducted De novo assembly using a software ABySS [61] according to the pipeline of Saunders et al. 2014 [62]. We evaluated N50 of the assembled sequences that were created following different $\mathrm{K}$-mer values and chose the sequences having the highest N50 value for the further 
analyses. To assess quality of the assembled sequences, we used the program CEGMA [30]. We predicted genes from the assembled sequences based on ab-initio and empirical methods. We estimated gene structure based on the published transcript sequences version 8 of $M$. oryzae 70-15 strain (Broad institute) and known $M$. oryza effectors [41], and then created an annotation files as general feature format (gff) using a software exonerate version 2.0 [63]. Genes of each assembled sequence of $M$. oryzae and M. grisea isolates were predicted using a software AUGUSTUS version 2.7 [64] after the training based on the created annotation files. SignalP4.0 was performed to predict secreted proteins [65]. Functional domains of the predicted genes were inferred using HMM-based search against Pfam [39]. BLASTN was performed using the predicted proteins as query against Swiss-Prot. Blast2GO [66] was run using the outputs of the BLASTN [67] as input files to clarify descriptions of proteins encoded by the predicted genes. To reveal orthologous relationship for each gene model, OrthoMCL was performed with $95 \%$ identity cutoff [33]. We used third codon positions of 3257 single copy genes having start and stop codons for the construction of phylogenetic trees.

To identify SNPs and indels among Magnaporthe species, paired-end short reads from each species were aligned with BWA [68] to the 70-15 strain (MG8) reference sequence and the assembled genome sequences of the five crop infecting M. oryzae isolates. Alignment files were converted to SAM files using SAMtools version 0.1.8 [69]. To remove low quality reads, modify misalignment reads and to detect reliable SNPs and short indels, the SAM files are applied to the filter pipeline "Coval" [70]. This pipeline reduces misalignments around short indels, and removes reads with errors arising from low phred-like quality scores and paired-end reads of an insert size longer than expected from the preparation of libraries. We called homozygous SNPs with default setting of Coval. We ignored heterozygous SNPs because we extracted DNAs from haploid mycelia of Magnaporthe species. Most of heterozygous SNPs could be caused by misalignments or reads that are aligned on multiple genomic regions.

To detect loss of genes in tested isolates from the reference genome, we estimate breadth coverage over a gene based on the alignments according to Raffaele et al. 2010 [36]. When short reads were aligned to their own assembled genome sequence using BWA, the smallest value of the breadth coverage was from $56 \%$ (Ina168 isolate) to $78 \%$ (Z2-1 isolate). If the breadth coverage is more than $50 \%$, the tested gene was judged presence on these minimum coverage values. If the breadth coverage is equal to zero percent, the tested gene was judged absence. The other values of breadth coverage were regarded as ambiguity and were not used for evaluating presence and absence polymorphisms. To clarify the pattern of presence and absence polymorphisms among the crop infecting $M$. oryzae isolates, Venn diagram was created using an online diagram software Cleately (Cinergix, Melbourne, Australia).

\section{Construction of phylogeny and estimation of nucleotide substitutions and polymorphisms}

The maximum likelihood trees were constructed using the RAxML software [71]. Consensus sequences for each gene were created based on SNPs and pileup format of SAMtools. We regarded positions where no short reads were aligned as unknown nucleotides. When nucleotides were different from those of the reference genome but were not regarded as homozygous SNPs by Coval, we also treated them as unknown nucleotides. Synonymous divergence $(\mathrm{dS})$ and nonsynonymous divergence $(\mathrm{dN})$, and omega $(\mathrm{dN} / \mathrm{dS})$ between $M$. oryzae and M. grisea isolates were estimated based on the consensus sequences using yn00 [38], which is an ad hoc counting method. When unknown bases were occupied over $80 \%$ of total length of the consensus sequence, we did not include this consensus sequence for calculation of $d N, d S$ and omega. Thresholds for detecting outliers of $\mathrm{dN} / \mathrm{dS}$ were calculated by $1.5 \times$ interquartile range $+3^{\text {rd }}$ quartile of $\mathrm{dN} / \mathrm{dS}$ in each pairwise comparison between isolates. If a threshold value was $<1.5,1.5$ was used as the threshold value.

To examine level and pattern of nucleotide polymorphisms within Oryza isolates and Setaria isolates, we calculated nucleotide diversity $\pi[72]$ and the number of net nucleotide substitutions per site between populations $\mathrm{Da}$ [72] as a parameter of genetic distance between Oryza isolates and Setaria isolates by using the Bioperl libraries. Sliding window analysis of $\pi$, and $\mathrm{Da}$ was performed with the $500 \mathrm{Kbp}$ widow size and $50 \mathrm{Kbp}$ step size. $\mathrm{R}$, a programming language for statistical computing, was used for visualization of the sliding window analysis. To test the neutral hypothesis of molecular evolution, McDonald and Kreitman test [55] was carried out according to the method of DNASP [73].

\section{Linkage of transposable elements and genetic variations}

To test relationship between transposable elements (TEs) and genetic variations, positions of TEs were identified. We performed BLASTN [67] using known M. oryzae transposable TEs (Additional file 1: Table S6) as query and the 70-15 genome sequence as database with the default setting. The aligned genome regions were regarded as the positions of TEs. We calculated physical distance from TEs and $5^{\prime}$ or $3^{\prime}$ end of tested genes. To test whether genes showing genetic variations within Oryza isolates and Setaria isolates linked to TEs or not, 
we used positions of TEs on the supercontigs of $M$. oryzae $70-15$ strain.

\section{Ethics}

This article does not contain any studies with human participants or animals performed by any of the authors.

\section{Consent to publish}

Not applicable.

\section{Availability of data and materials}

Nucleotide sequence data reported are available in the DDBJ Sequenced Read Archive under the accession numbers DRR059884-DRR059895. The assembled genome sequences of $M$. oryzae isolates Ina168, GFSI1-7-2, $\mathrm{Br} 48, \mathrm{Br} 58$ and Z2-1 and M. grisea isolate Dig41 have been deposited to DDBJ Sequenced Read Archive under the accession numbers DRZ007458, DRZ007459, DRZ007460, DRZ007461, DRZ007462, and DRZ007468, respectively. The annotation files of $M$. oryzae isolates Ina168, GFSI1-7-2, Br48, Br58 and Z2-1 and M. grisea isolate Dig41 are available in the DDBJ Sequenced Read Archive under the accession numbers DRZ007463, DRZ007464, DRZ007465, DRZ007466, DRZ007467 and DRZ007469, respectively.

\section{Additional files}

Additional file 1: This file contains Supplemental Tables S1 to S6 and their legends. (PDF $130 \mathrm{~kb}$ )

Additional file 2: This sheet includes information about annotation, presence/absence polymorphisms and dN/dS for each of the gene models. (XLSX $12390 \mathrm{~kb}$ )

Additional file 3: This file contains Supplemental Figures S1 to S8 and their legends. (PDF 1373 kb)

\section{Abbreviations \\ CEGs: core eukaryotic protein genes; Da: the number of net nucleotide substitutions per site between populations; dN/dS: the ratio of nonsynonymous substitutions to synonymous substitutions; dS: synonymous divergence; $\mathrm{dN}$ : nonsynonymous divergence; HsbA: hydrophobic surface binding protein A; LTR: Iong terminal repeat; LysM: Iysin motif; MK test: McDonald and Kreitman's test; ML: maximum likelihood; PAMP: pathogen-associated molecular pattern; SIp1: Secreted LysM protein 1; SNPS: single nucleotide polymorphisms; TEs: transposable elements; $\pi$ : nucleotide diversity.}

\section{Competing interests}

The authors declare that they have no competing interests.

\section{Authors' contribution}

KY, RT and SK (S. Kamoun) designed the whole project. DGOS, KY, RT and SK (S. Kamoun) wrote the manuscript. CM, HS, IC and KY carried out experiments. DGOS, KY, LCM, SK (S. Kosugi) and SN designed pipelines of genome analysis. $\mathrm{KY}, \mathrm{SN}$ and $\mathrm{YI}$ performed genome analysis. IC and YT provided materials. All authors have read and approved the final version of the manuscript.

\section{Acknowledgements}

Computations for the sequencing analyses were performed on the NIG supercomputer at the ROIS National Institute of Genetics, Japan. M. oryzae KANSV1-4-1, SA05-43 and SA05-144 isolates were provided by National Institute of Agrobiological Sciences Genebank, Japan.

\section{Funding}

This work was supported by the 'Program for Promotion of Basic Research Activities for Inovative Biosciences (PROBRAIN)' (Japan), 'The Ministry of Agriculture, Forestry, and Fisheries of Japan (Genomics for Agricultural Innovation PMI-0010)', the Gatsby Charitable Foundation and the Biotechnology and Biological Sciences Research Council (BBSRC). DS is supported through a Biotechnology and Biological Sciences Research Council (BBSRC) fellowship in computational Biology. RT was supported by the Programme for Promotion of Basic and Applied Researches for Innovations in Bio-oriented Industry, Grant-inaid for MEXT (Scientific Research on Innovative Areas 23113009) and JSPS KAKENHI (Grant Nos. 24248004, 15H05779).

\section{Author details \\ ${ }^{1}$ Iwate Biotechnology Research Center, Kitakami, Iwate, Japan. ${ }^{2}$ The Sainsbury Laboratory, Norwich Research Park, Norwich, UK. ${ }^{3}$ Graduate School of Agricultural Science, Kobe University, Kobe, Japan. ${ }^{4}$ The Genome Analysis Centre, Norwich Research Park, Noriwich, UK. 5John Innes Centre, Norwich Research Park, Norwich, UK. ${ }^{6}$ Department of Plant Pathology, Indian River Research and Education Center, University of Florida, Fort Pierce, USA.}

Received: 26 December 2015 Accepted: 5 May 2016

Published online: 18 May 2016

\section{References}

1. Kato H, Yamamoto M, Yamaguchi-Ozaki T, Kadouchi H, Iwamoto Y, Nakayashiki $\mathrm{H}$, et al. Pathogenicity, Mating Ability and DNA Restriction Fragment Length Polymorphisms of Pyricularia Populations Isolated from Gramineae, Bambusideae and Zingiberaceae Plants. J Gen Plant Pathol. 2000;66:30-47.

2. Dean R, Van Kan JAL, Pretorius ZA, Hammond-Kosack KE, Di Pietro A, Spanu PD, et al. The Top 10 fungal pathogens in molecular plant pathology. Mol Plant Pathol. 2012;13:414-30.

3. Talbot NJ. On the trail of a cereal killer: Exploring the biology of Magnaporthe grisea. Annu Rev Microbiol. 2003;57:177-202.

4. Couch BC, Fudal I, Lebrun M-H, Tharreau D, Valent B, van Kim P, et al. Origins of host-specific populations of the blast pathogen Magnaporthe oryzae in crop domestication with subsequent expansion of pandemic clones on rice and weeds of rice. Genetics. 2005;170:613-30.

5. Hirata K, Kusaba M, Chuma I, Osue J. Speciation in Pyricularia inferred from multilocus phylogenetic analysis. Mycol Res. 2007;111:799-808.

6. Fisher MC, Henk DA, Briggs CJ, Brownstein JS, Madoff LC, McCraw SL, et al. Emerging fungal threats to animal, plant and ecosystem health. Nature. 2012:484:186-94.

7. Kupferschmidt K. Attack of the Clones. Science. 2012;337:636-8.

8. Hayashi N, Li C, Li J, Naito H. In vitro production on rice plants of perithecia of Magnaporthe grisea from Yunnan, China. Mycol Res. 1997;101:1308-10.

9. Kumar J, Nelson R, Zeigler R. Population Structure and Dynamics of Magnaporthe grisea in the Indian Himalayas. Genetics. 1999;152:971-84.

10. Saleh D, Xu P, Shen Y, Li C, Adreit H, Milazzo J, Ravigné V, Bazin E, Nottéghem J-L, Fournier E, Tharreau D. Sex at the origin: an Asian population of the rice blast fungus, Magnaporthe oryzae, reproduces sexually. Mol Ecol. 2012:21:1330-44.

11. Muller HJ. The relation of recombination to mutational advance. Mutat Res. 1964;106:2-9.

12. Seidl MF, Thomma BPHJ. Sex or no sex: evolutionary adaptation occurs regardless. BioEssays. 2014;36:335-45.

13. De Jonge $R$, Bolton MD, Kombrink A, Van Den Berg GCM, Yadeta KA, Thomma BPHJ. Extensive chromosomal reshuffling drives evolution of virulence in an asexual pathogen. Genome Res. 2013;23:1271-82.

14. Haas BJ, Kamoun S, Zody MC, Jiang RHY, Handsaker RE, Cano LM, et al. Genome sequence and analysis of the Irish potato famine pathogen Phytophthora infestans. Nature. 2009;461:393-8.

15. Chuma I, Isobe C, Hotta Y, Ibaragi K, Futamata N, Kusaba M, et al. Multiple translocation of the AVR-Pita effector gene among chromosomes of the rice blast fungus Magnaporthe oryzae and related species. PLoS Pathog. 2011;7, e1002147.

16. Wicker T, Oberhaensli S, Parlange F, Buchmann JP, Shatalina M, Roffler S, et al. The wheat powdery mildew genome shows the unique evolution of an obligate biotroph. Nat Genet. 2013;45:1092-6.

17. Raffaele S, Kamoun S. Genome evolution in filamentous plant pathogens: why bigger can be better. Nat Rev Microbiol. 2012;10:417-30. 
18. Xue M, Yang J, Li Z, Hu S, Yao N, Dean RA, et al. Comparative analysis of the genomes of two field isolates of the rice blast fungus Magnaporthe oryzae. PLoS Genet. 2012;8, e1002869.

19. Yoshida K, Saitoh H, Fujisawa S, Kanzaki H, Matsumura H, Yoshida K, et al. Association genetics reveals three novel avirulence genes from the rice blast fungal pathogen Magnaporthe oryzae. Plant Cell. 2009;21: 1573-91.

20. Huang J, Si W, Deng Q, Li P, Yang S. Rapid evolution of avirulence genes in rice blast fungus Magnaporthe oryzae. BMC Genet. 2014;15:45.

21. Kanzaki H, Yoshida K, Saitoh H, Fujisaki K, Hirabuchi A, Alaux L, et al. Arms race co-evolution of Magnaporthe oryzae AVR-Pik and rice Pik genes driven by their physical interactions. Plant J. 2012;72:894-907.

22. Magbool A, Saitoh H, Franceschetti M, Stevenson C, Uemura A, Kanzaki H, et al. Structural basis of pathogen recognition by an integrated HMA domain in a plant NLR immune receptor. Elife. 2015;4, e08709.

23. Urashima A, Igarashi $\mathrm{S}$, Kato $\mathrm{H}$. Host range, mating type, and fertility of Pyricularia grisea from wheat in Brazil. Plant Dis. 1993;77:1211-6.

24. Saleh D, Milazzo J, Adreit H, Fournier E, Tharreau D. South-East Asia is the center of origin, diversity and dispersion of the rice blast fungus, Magnaporthe oryzae. New Phytol. 2013;201:1440-56.

25. Yamagashira A, Iwai C, Misaka M, Hirata K, Fujita Y, Tosa $Y$, et al. Taxonomic characterization of Pyricularia isolates from green foxtail and giant foxtail, wild foxtails in Japan. J Gen Plant Pathol. 2008;74:230-41.

26. Murakami J, Tosa Y, Kataoka T. Analysis of host species specificity of Magnaporthe grisea toward wheat using a genetic cross between isolates from wheat and foxtail millet. Phytopathology. 2000;90:1060-7.

27. Takabayashi N, Tosa Y, Oh H, Mayama S. A gene-for-gene relationship underlying the species-specific parasitism of Avena/Triticum isolates of Magnaporthe grisea on wheat cultivars. Phytopathology. 2002;92:1182-8.

28. Tosa Y, Tamba H, Tanaka K, Mayama S. Genetic analysis of host species specificity of Magnaporthe oryzae isolates from rice and wheat. Phytopathology. 2006:96:480-4.

29. Chiapello H, Mallet L, Guérin C, Aguileta G, Amselem J, Kroj T, et al. Deciphering Genome Content and Evolutionary Relationships of Isolates from the Fungus Magnaporthe oryzae Attacking Different Host Plants. Genome Biol Evol. 2015;7:2896-912

30. Parra G, Bradnam K, Korf I. CEGMA: a pipeline to accurately annotate core genes in eukaryotic genomes. Bioinformatics. 2007;23:1061-7.

31. Cantu D, Segovia V, MacLean D, Bayles R, Chen X, Kamoun S, et al. Genome analyses of the wheat yellow (stripe) rust pathogen Puccinia striiformis $\mathrm{f}$. sp. tritici reveal polymorphic and haustorial expressed secreted proteins as candidate effectors. BMC Genomics. 2013;14:270.

32. Sharma R, Mishra B, Runge $F$, Thines $M$. Gene loss rather than gene gain is associated with a host jump from Monocots to Dicots in the Smut Fungus Melanopsichium pennsylvanicum. Genome Biol Evol. 2014;6:2034-49.

33. Li L, Stoeckert CJ, Roos DS. OrthoMCL: identification of ortholog groups for eukaryotic genomes. Genome Res. 2003;13:2178-89.

34. Couch BC, Kohn LM. A multilocus gene genealogy concordant with host preference indicates segregation of a new species, Magnaporthe oryzae, from M. grisea. Mycologia. 2002;94:683-93.

35. Klaubauf S, Tharreau D, Fournier E, Groenewald JZ, Crous PW, de Vries RP, et al. Resolving the polyphyletic nature of Pyricularia (Pyriculariaceae). Stud Mycol. 2014;79:85-120

36. Raffaele S, Farrer RA, Cano LM, Studholme DJ, MacLean D, Thines M, et al. Genome evolution following host jumps in the Irish potato famine pathogen lineage. Science. 2010;330:1540-3.

37. Dong S, Stam R, Cano LM, Song J, Sklenar J, Yoshida K, et al. Effector specialization in a lineage of the Irish potato famine pathogen. Science. 2014:343:552-5.

38. Yang Z. PAML 4: phylogenetic analysis by maximum likelihood. Mol Biol Evol. 2007;24:1586-91.

39. Finn RD, Mistry J, Tate J, Coggill P, Heger A, Pollington JE, et al. The Pfam protein families database. Nucleic Acids Res. 2010;38:D211-22.

40. Soanes DM, Chakrabarti A, Paszkiewicz KH, Dawe AL, Talbot NJ. Genomewide transcriptional profiling of appressorium development by the rice blast fungus Magnaporthe oryzae. PLoS Pathog. 2012;8, e1002514.

41. Giraldo MC, Valent B. Filamentous plant pathogen effectors in action. Nat Rev Microbiol. 2013;11:800-14.

42. Sweigard JA, Carroll AM, Kang S, Farrall L, Chumley FG, Valent B. Identification, cloning, and characterization of PWL2, a gene for host species specificity in the rice blast fungus. Plant Cell. 1995;7:1221-33.
43. Kang S, Sweigard JA, Valent B. The PWL host specifity gene family in the blast fungus Magnaporthe grisea. Mol Plant Microbe Interact. 1995;8:939-48.

44. Orbach $M$, Farrall $L$. A telomeric avirulence gene determines efficacy for the rice blast resistance gene Pi-ta. Plant Cell. 2000;12:2019-32.

45. Khang C, Park S. Genome organization and evolution of the AVR-Pita avirulence gene family in the Magnaporthe grisea species complex. Mol Plant-Microbe Interact. 2008;21:658-70.

46. Li W, Wang B, Wu J, Lu G. The Magnaporthe oryzae avirulence gene AvrPiz-t encodes a predicted secreted protein that triggers the immunity in rice mediated by the blast resistance gene Piz-t. Mol Plant-Microbe Interact. 2009;22:411-20.

47. Cesari S, Thilliez G, Ribot C, Chalvon V, Michel C, Jauneau A, et al. The rice resistance protein pair RGA4/RGA5 recognizes the Magnaporthe oryzae effectors AVR-Pia and AVR1-CO39 by direct binding. Plant Cell. 2013;25: 1463-81.

48. Mosquera G, Giraldo MC, Khang CH, Coughlan S, Valent B. Interaction transcriptome analysis identifies Magnaporthe oryzae BAS1-4 as Biotrophyassociated secreted proteins in rice blast disease. Plant Cell. 2009;21:1273-90.

49. Giraldo MC, Dagdas YF, Gupta YK, Mentlak TA, Yi M, Martinez-Rocha AL, et al. Two distinct secretion systems facilitate tissue invasion by the rice blast fungus Magnaporthe oryzae. Nat Commun. 2013;4:1-12.

50. Wu J, Kou Y, Bao J, Li Y, Tang M, Zhu X, et al. Comparative genomics identifies the Magnaporthe oryzae avirulence effector AvrPi9 that triggers Pi9-mediated blast resistance in rice. New Phytol. 2015;206:1463-75.

51. Zhang S, Wang L, Wu W, He L, Yang X, Pan Q. Function and evolution of Magnaporthe oryzae avirulence gene AvrPib responding to the rice blast resistance gene Pib. Sci Rep. 2015:5:11642.

52. Mentlak TA, Kombrink A, Shinya T, Ryder LS, Otomo I, Saitoh H, et al. Effector-mediated suppression of chitin-triggered immunity by magnaporthe oryzae is necessary for rice blast disease. Plant Cell. 2012;24: 322-35

53. Saitoh H, Fujisawa S, Mitsuoka C, Ito A, Hirabuchi A, Ikeda K, et al. Largescale gene disruption in Magnaporthe oryzae identifies MC69, a secreted protein required for infection by monocot and dicot fungal pathogens. PLoS Pathog. 2012;8, e1002711.

54. Böhnert HU, Fudal I, Dioh W, Tharreau D, Notteghem J-L, Lebrun M-H. A putative polyketide synthase/peptide synthetase from Magnaporthe grisea signals pathogen attack to resistant rice. Plant Cell. 2004;16:2499-513.

55. McDonald $\mathrm{JH}$, Kreitman M. Adaptive protein evolution at the Adh locus in Drosophila. Nature. 1991;351:652-4.

56. Robberecht C, Voet T, Zamani Esteki M, Nowakowska BA, Vermeesch JR. Nonallelic homologous recombination between retrotransposable elements is a driver of de novo unbalanced translocations. Genome Res. 2013;23: 411-8.

57. Jones JDG, Dangl JL. The plant immune system. Nature. 2006;444:323-9.

58. De Guillen K, Ortiz-Vallejo D, Gracy J, Fournier E, Kroj T, Padilla A. Structure analysis uncovers a highly diverse but structurally conserved effector family in phytopathogenic fungi. PLoS Pathog. 2015;11, e1005228.

59. Sharma S, Sharma S, Hirabuchi A, Yoshida K, Fujisaki K, Ito A, et al. Deployment of the Burkholderia glumae type III secretion system as an efficient tool for translocating pathogen effectors to monocot cells. Plant J. 2013;74:701-12.

60. Martin M. Cutadapt removes adapter sequences from high-throughput sequencing reads. EMBnet J. 2011;17:10-2.

61. Simpson JT, Wong K, Jackman SD, Schein JE, Jones SJM, Birol I. ABySS: a parallel assembler for short read sequence data. Genome Res. 2009;19:1117-23.

62. Saunders D, Yoshida K, Sambles C, Glover R, Clavijo B, Corpas M, et al. Crowdsourced analysis of ash and ash dieback through the Open Ash Dieback project: A year 1 report on datasets and analyses contributed by a self-organising community. bioRxiv. 2014. doi:10.1101/004564.

63. Slater GSC, Birney E. Automated generation of heuristics for biological sequence comparison. BMC Bioinformatics. 2005;6:31.

64. Stanke M, Waack S. Gene prediction with a hidden Markov model and a new intron submodel. Bioinformatics. 2003;19:ii215-5.

65. Petersen TN, Brunak S, von Heijne G, Nielsen H. SignalP 4.0: discriminating signal peptides from transmembrane regions. Nat Methods. 2011;8:785-6.

66. Conesa A, Götz S, García-Gómez JM, Terol J, Talón M, Robles M. Blast2GO: a universal tool for annotation, visualization and analysis in functional genomics research. Bioinformatics. 2005;21:3674-6.

67. Altschul S, Gish W, Miller W. Basic local alignment search tool. J Mol Biol. 1990;215:403-10. 
68. Li H, Durbin R. Fast and accurate short read alignment with BurrowsWheeler transform. Bioinformatics. 2009;25:1754-60.

69. Li H, Handsaker B, Wysoker A, Fennell T, Ruan J, Homer N, et al. The Sequence Alignment/Map format and SAMtools. Bioinformatics. 2009;25: 2078-9.

70. Kosugi S, Natsume S, Yoshida K, MacLean D, Cano L, Kamoun S, et al. Coval: improving alignment quality and variant calling accuracy for nextgeneration sequencing data. PLoS One. 2013;8, e75402.

71. Stamatakis A. RAxML version 8: a tool for phylogenetic analysis and postanalysis of large phylogenies. Bioinformatics. 2014;30:1312-3.

72. Nei M. Molecular Evolutionary Genetics. New York: Columbia University Press; 1987.

73. Rozas J, Sanchez-DelBarrio JC, Messeguer X, Rozas R. DnaSP, DNA polymorphism analyses by the coalescent and other methods. Bioinformatics. 2003;19:2496-7.

Submit your next manuscript to BioMed Central and we will help you at every step:

- We accept pre-submission inquiries

- Our selector tool helps you to find the most relevant journal

- We provide round the clock customer support

- Convenient online submission

- Thorough peer review

- Inclusion in PubMed and all major indexing services

- Maximum visibility for your research

Submit your manuscript at www.biomedcentral.com/submit
Biomed Central 